\title{
Modeling and Observation of Nonlinear Damping in Dissipation-Diluted Nanomechanical Resonators
}

\author{
Letizia Catalini®, Massimiliano Rossi®, ${ }^{*}$ Eric C. Langman, and Albert Schliesser® ${ }^{\dagger}$ \\ Niels Bohr Institute, University of Copenhagen, Blegdamsvej 17, 2100 Copenhagen, Denmark and \\ Center for Hybrid Quantum Networks (Hy-Q), Niels Bohr Institute, University of Copenhagen, 2100 Copenhagen, Denmark
}

(Received 14 January 2021; accepted 29 March 2021; published 28 April 2021)

\begin{abstract}
Dissipation dilution enables extremely low linear loss in stressed, high aspect ratio nanomechanical resonators, such as strings or membranes. Here, we report on the observation and theoretical modeling of nonlinear dissipation in such structures. We introduce an analytical model based on von Kármán theory, which can be numerically evaluated using finite-element models for arbitrary geometries. We use this approach to predict nonlinear loss and (Duffing) frequency shift in ultracoherent phononic membrane resonators. A set of systematic measurements with silicon nitride membranes shows good agreement with the model for low-order soft-clamped modes. Our analysis also reveals quantitative connections between these nonlinearities and dissipation dilution. This is of interest for future device design and can provide important insight when diagnosing the performance of dissipation dilution in an experimental setting.
\end{abstract}

DOI: 10.1103/PhysRevLett.126.174101

Introduction.--In recent decades, micro- and nanomechanical systems have attracted widespread interest in science and technology [1,2]. They constitute outstanding sensors for force [3], mass [4], radiation [5], and temperature [6], to name just a few examples. Simultaneously, they are promising building blocks for future quantum technologies, such as microwave- or spin-to-optical quantum transducers [7,8] or quantum memories [9].

Low thermomechanical noise and correspondingly long mechanical coherence times are crucial for these applications and are typically limited by energy dissipation from the mode of interest. Considerable effort has therefore gone into designing mechanical resonators with minimal dissipation, leading to great advances in recent decades. Beyond mitigating all external losses, e.g., due to the surrounding gas or the device substrate, important progress was made in suppressing loss to internal degrees of freedom, such as two-level systems. By storing the majority of the mechanical mode's energy in a lossless potential, dissipation dilution [10] has emerged as a successful strategy in this endeavour. It can be utilized in highly stressed nanomechanical string and membrane systems, where the elongation energy assumes the role of the lossless potential [11-15].

We have recently introduced an extension of this approach-soft clamping-to engineer mechanical

Published by the American Physical Society under the terms of the Creative Commons Attribution 4.0 International license. Further distribution of this work must maintain attribution to the author(s) and the published article's title, journal citation, and DOI. resonance modes particularly conducive to dissipation dilution in phononic crystal membranes [16].

Soft clamping has allowed realizing nanomechanical resonators with the highest $Q$ factors $\left(>10^{8}\right)$ and $Q f$ products $\left(>10^{15} \mathrm{~Hz}\right)$ yet observed at room temperature [16-19]. For the vast majority of mechanical systems, it is sufficient to consider dissipation in the linear regime, i.e., when the quality factor is independent of the displacement amplitude [1]. Some instances of nonlinear dissipation in nanomechanical systems have been reported, for example, in nanoresonators made from diamond [20], carbon nanotubes, and graphene sheets [21], but without providing a clear explanation as to the origin of this effect. Here, we investigate nonlinear effects in soft-clamped membrane resonators with very high $\mathrm{Q}$ factors. Whereas the Duffing frequency shift has been observed in stressed nanomechanical resonators before [22-24], we focus on nonlinear damping here [19,25].

Starting from a full 3D model (von Kármán theory), we derive analytical expressions for both the Duffing frequency shift and nonlinear damping, similar to what has been derived for a string [2]. This analysis furthermore reveals strong connections to dissipation dilution, both being linked to geometric nonlinearities.

A series of systematic experiments yields good quantitative agreement with the model for low-order soft-clamped modes. We thereby establish not only a means to quantitatively predict nonlinear losses-as relevant, e.g., for parametric sensing protocols [26] — but also introduce a new experimental tool for assessing dissipation dilution.

Model.-We describe the motion of a thin membrane, of thickness $h$. The $x y$ plane coincides with the one of the undeformed membrane. 
The displacement of the mass element located at position $\mathbf{r}(x, y, z)$ is quantified by the vector $u_{i}$, where Latin indexes represent the three directions $x, y, z$. The membrane's deformation due to the motion of the mass elements is expressed by the strain tensor $\varepsilon_{i j}=$ $\left(\partial_{j} u_{i}+\partial_{i} u_{j}+\partial_{i} u_{z} \partial_{j} u_{z}\right) / 2$. The deformation induces stresses within the structure, described by the stress tensor $\sigma_{i j}$. We consider elastic materials, for which the induced stresses are linear in the strain tensor and Hooke's law holds [27]. For thin membranes with no external loads, stress components associated with the $z$ direction are negligible, i.e., $\sigma_{i z}=0$. The membrane's displacement can then be decomposed into the out-of-plane displacement $u_{z}(x, y, z) \equiv w(x, y)$ and the in-plane displacement $u_{\alpha}(x, y, z)=v_{\alpha}(x, y)-z \partial_{\alpha} w(x, y)$, where Greek indexes represent the in-plane coordinates $x$ and $y$, and $v_{\alpha}$ is the inplane displacement. For amplitudes relevant to this work, the in-plane displacement components are negligible with respect to the out-of-plane components [confirmed with finite element method (FEM) simulations]. Therefore, we apply the so-called out-of-plane approximation and neglect them [28]. Furthermore, our model includes a static inplane deformation $\varepsilon_{0}(x, y)$ representing the static strain required for dissipation dilution. The shear components of the stress tensor $\left(\sigma_{0}\right)_{x y}$ are negligible in the structures considered. Within these approximations, we can write the strain and stress tensors, according to von Kármán theory $[27,28]$, as

$$
\begin{gathered}
\varepsilon_{\alpha \beta}=\varepsilon_{0} \delta_{\alpha \beta}-z \partial_{\alpha \beta} w+\frac{1}{2} \partial_{\alpha} w \partial_{\beta} w, \\
\sigma_{\alpha \beta}=\frac{E}{1-\nu^{2}}\left[(1-\nu) \varepsilon_{\alpha \beta}+\nu \varepsilon_{\gamma \gamma} \delta_{\alpha \beta}\right],
\end{gathered}
$$

where $E$ is the Young's modulus, $\nu$ is the Poisson's ratio, $\delta_{\alpha \beta}$ is the Kronecker delta, and repeated Greek indexes are summed over.

To introduce dissipation, we assume a time delay $\tau$ in the stress-strain relation, as a phenomenological model for microscopic relaxation processes intrinsic to the resonator material [2]. For small and constant time delay (relative to mechanical period), the stress tensor can be approximated as $\sigma(t)=H[\varepsilon(t+\tau)] \approx H[\varepsilon(t)]+\tau H[\dot{\varepsilon}(t)]$, where $H$ is a linear functional expressing Hooke's law and $\dot{\varepsilon}$ stands for the time derivative of the stress tensor. The dissipation arises from the additional term proportional to $\tau$. The equation of motion for out-of-plane displacement is

$$
\begin{gathered}
\rho h \ddot{w}-\partial_{\alpha \beta} M_{\alpha \beta}-\partial_{\beta}\left(N_{\alpha \beta} \partial_{\alpha} w\right)=0, \\
\partial_{\beta} N_{\alpha \beta}=0,
\end{gathered}
$$

where the stress resultants $N_{\alpha \beta}$ and $M_{\alpha \beta}$ are the shear force components and bending momenta, respectively. They are given by $N_{\alpha \beta}=\int \sigma_{\alpha \beta} d z$ and $M_{\alpha \beta}=\int z \sigma_{\alpha \beta} d z$, where each integral is performed over the membrane thickness. As both stress resultants contain terms proportional to the time delay $\tau$, they generate both linear and nonlinear dissipative processes, as we shall see. Typically, Eqs. (3) are difficult to solve due to nonlinearity in the displacement $w$. For small $w$, we neglect nonlinear terms and retrieve a solvable linear equation, yielding a set of normal modes $w_{\eta}(x, y, t)=\phi_{\eta}(x, y) u_{\mu}(t) \delta_{\eta \mu}$, where $\eta$ indexes different vibrational modes and we have separated the time-dependent mode amplitude $u_{\mu}(t)$ from the dimensionless transverse spatial profile $\phi_{\eta}(x, y)$. We use this set of transverse modes as a basis to expand a solution of the full nonlinear equation of motion; that is, $w(x, y, t)=\phi_{\eta}(x, y) u_{\eta}(t)$. We insert this ansatz in Eq. (3), then project it into a single transverse mode $\phi_{i}$ by applying the Galerkin method together with a single mode approximation that neglects intermodal coupling [29]. Finally, we obtain the following effective nonlinear equation for the temporal mode $u_{i}$ :

$$
\ddot{u}_{i}+\Gamma_{i} \dot{u}_{i}+\gamma_{i}^{\mathrm{nl}} u_{i}^{2} \dot{u}_{i}+\Omega_{i}^{2} u_{i}+\beta_{i} u_{i}^{3}=0,
$$

which describes a damped Duffing resonator [22,23], including a nonlinear damping term [19,25,30-32]. The effective parameters in Eq. (4) are defined as

$$
\begin{aligned}
& \Omega_{i}^{2}=m_{\mathrm{eff}}^{-1} \int \phi_{i}\left(D \partial_{\alpha \alpha \beta \beta} \phi_{i}-h \sigma_{0} \partial_{\alpha \alpha} \phi_{i}\right) d A \\
& \beta_{i}=\frac{k_{1}}{2} \int \phi_{i}\left(\partial_{\alpha \beta} \phi_{i} \partial_{\alpha} \phi_{i} \partial_{\beta} \phi_{i}+k_{2} \partial_{\alpha \alpha} \phi_{i} \partial_{\beta} \phi_{i} \partial_{\beta} \phi_{i}\right) d A \\
& \Gamma_{i}=\tau D m_{\mathrm{eff}}^{-1} \int \phi_{i} \partial_{\alpha \alpha \beta \beta} \phi_{i} d A
\end{aligned}
$$

$\gamma_{i}^{\mathrm{nl}}=k_{1} \tau \int \phi_{i}\left(\partial_{\alpha \beta} \phi_{i} \partial_{\alpha} \phi_{i} \partial_{\beta} \phi_{i}+k_{2} \partial_{\alpha \alpha} \phi_{i} \partial_{\beta} \phi_{i} \partial_{\beta} \phi_{i}\right) d A$,

where the integrals extend over the whole membrane surface, $m_{\text {eff }}=\rho h \int \phi_{i}^{2} d A$ is the effective mass, $D=$ $E h^{3} /\left[12\left(1-\nu^{2}\right)\right]$ is the flexural rigidity, and we have introduced the constants $k_{1}=-h E /\left[m_{\mathrm{eff}}\left(1-\nu^{2}\right)\right]$ and $k_{2}=\nu /(1-\nu)$. Notably, the two nonlinear terms are purely geometric effects and they are not introduced by the material itself.

As expected, we find both the linear $\left(\Gamma_{i}\right)$ and nonlinear $\left(\gamma_{i}^{\mathrm{nl}}\right)$ dissipation proportional to the lag time $\tau$. In the context of dissipation dilution, the linear dissipation is commonly expressed as

$$
\Gamma_{i}=\frac{1}{D_{Q, i}} \frac{\Omega_{i}}{Q_{\text {intr }}},
$$

where $D_{Q, i} \gg 1$ is the dissipation dilution factor, determined by the geometry of mode $i[10-16]$. The resonator's 
material properties enter via the intrinsic quality factor $Q_{\text {intr }}=\left(\Omega_{i} \tau\right)^{-1}$, which we use interchangeably with the material's loss angle $\theta_{\text {lin }}=Q_{\text {intr. }}^{-1}$. In dissipation-diluted devices, one expects to measure enhanced (linear) quality factors

$$
Q_{\text {meas }}:=\frac{\Omega_{i}}{\Gamma_{i}}=D_{Q, i} Q_{\text {intr }}=D_{Q, i} \theta_{\text {lin }}^{-1},
$$

compared to resonators made from the same material in absence of dissipation dilution (e.g., unstressed). In this setting, $D_{Q, i}$ and $Q_{\text {intr }}$ are not separately accessible through measurement.

Turning to nonlinear effects, we note that the Duffing frequency shift $\left(\beta_{i}\right)$ and nonlinear damping $\left(\gamma_{i}^{\mathrm{nl}}\right)$ depend on the mode pattern identically, yet their ratio depends on the lag time $\tau$ and thence on the intrinsic loss. We therefore introduce the nonlinear loss angle

$$
\theta_{\mathrm{nl}}:=\frac{\gamma_{i}^{\mathrm{nl}} \Omega_{i}}{2 \beta_{i}}
$$

which notably depends only on quantities that can be experimentally measured through large-amplitude excitation (see below). Importantly, Eqs. (5) suggest

$$
\theta_{\mathrm{nl}}=\Omega_{i} \tau=\theta_{\operatorname{lin}}
$$

which would imply access to the intrinsic linear damping through measurement of a device's nonlinear properties.

Experimental results.-Our experimental subjects are highly stressed $3.6 \times 3.6 \mathrm{~mm}$ soft-clamped $\mathrm{Si}_{3} \mathrm{~N}_{4}$ membrane resonators [16], shown in Fig. 1(b), operated at room temperature and pressures lower than $10^{-7}$ mbar to reduce gas damping to a negligible value. We expect intrinsic material dissipation to dominate and the theoretical framework derived above to apply since radiation losses of the vibrational defect modes are shielded by the honeycomb phononic crystal pattern. Nonlinear phenomena are present during free decay evolution as amplitude-dependent damping and shift of the mechanical resonance frequency. To observe these effects, we employ ringdown techniques and measure mechanical displacement with a fiber-based optical Mach-Zender interferometer [Fig. 1(a)]. After excitation to a large amplitude, we stop driving and monitor the displacement decaying with a heterodyne detector, from which we extract both the displacement amplitude $A_{i}$ and phase, thus the instantaneous frequency $\Omega_{i}^{\prime}$. The displacement amplitude and frequency evolve according to [19]

$$
\begin{aligned}
& \delta \Omega_{i}(t) \equiv \Omega_{i}-\Omega_{i}^{\prime}=\frac{3}{4} \omega_{i}^{s D} A_{i}^{2}(t), \\
& A_{i}(t)=\frac{A_{i_{0}} e^{-\frac{\Gamma_{i}}{2} t}}{\sqrt{1+\frac{\gamma_{i}^{\mathrm{nl}}}{4 \Gamma_{i}}} A_{i_{0}}^{2}\left(1-e^{-\Gamma_{i} t}\right)} .
\end{aligned}
$$
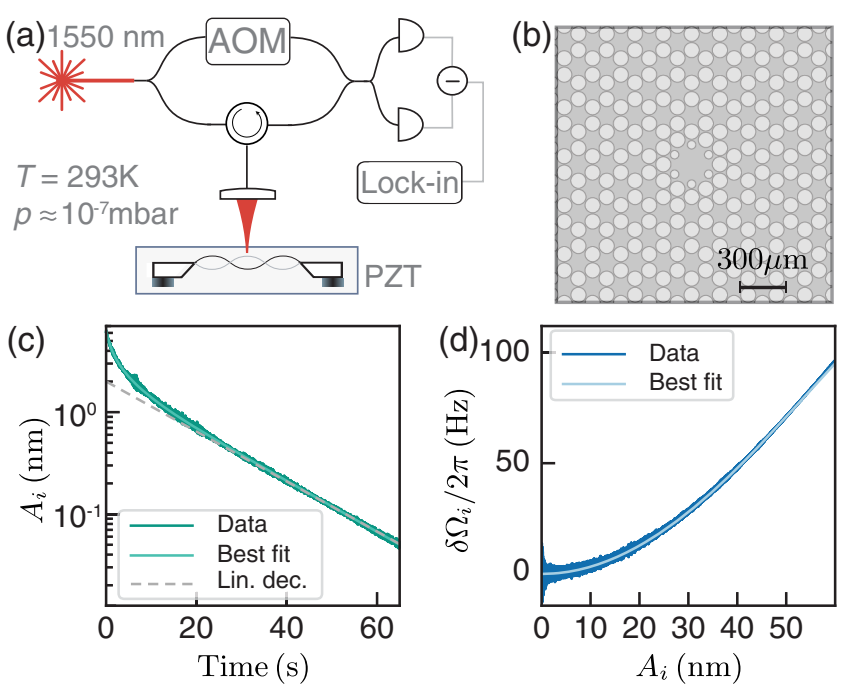

FIG. 1. (a) Optical interferometer for displacement measurement [acousto-optic modulator (AOM), piezoelectric actuator (PZT)]. The detection is realized with a heterodyne receiver. (b) Soft-clamped membrane pattern. (c) Nonlinear amplitude decay for 19-nm-thick membrane and corresponding fit. Linear exponential decay (gray) is extrapolated to highlight deviation arising from nonlinear damping. (d) Duffing frequency shift as a function of displacement amplitude and corresponding fit. The abscissa is the fit result from (c).

Equation (10) is the standard backbone equation [22,23], while Eq. (11) describes a nonexponential decay, induced by the nonlinear damping $[25,30]$. We have also introduced the Duffing shift per displacement, $\omega_{i}^{\mathrm{sD}}:=\beta_{i} /\left(2 \Omega_{i}\right)$, such that the nonlinear loss angle defined in Eq. (8) becomes $\theta_{\mathrm{nl}}^{-1}=4 \omega_{i}^{\mathrm{sD}} / \gamma_{i}^{\mathrm{nl}}$.

In Figs. 1(c) and 1(d), we show an example of nonlinear amplitude decay and frequency shift as a function of displacement amplitude. The nonlinear parameters are extracted from the best fit. Importantly, the values of both $\omega_{i}^{\mathrm{sD}}$ and $\gamma_{i}^{\mathrm{nl}}$ depend on the displacement calibration [19], however, their ratio $\theta_{\mathrm{nl}}$ is independent of that. Notice that deviation from linear decay starts appearing for displacement amplitudes comparable to the membrane thickness [32], as expected when the bending at the membrane edges has been eliminated (soft clamping). From the amplitude decay fit, we extract the linear damping rate $\Gamma_{i}$ and calculate $Q_{\text {meas }}$.

We perform five ringdown measurements on every individual mode, then average the results. Ringdowns are discarded if relative errors on fit parameters are greater than $10 \%$, using a $95 \%$ confidence interval. For each membrane, we characterize the nonlinear parameters of four defect modes lying in the band gap from 1.30 to $1.55 \mathrm{MHz}$ (see Supplemental Material [33]). Statistics are collected from 6 to 12 nominally identical membranes. Because of the presence of outliers within this ensemble, we evaluate and report the median and median absolute deviation as robust estimators of the ensemble statistics 
(a) $\Omega_{1}=2 \pi \times 1.38 \mathrm{MHz}$

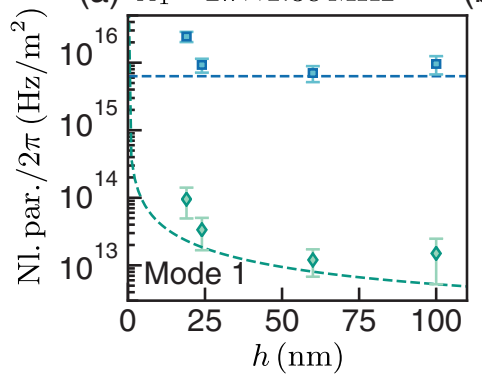

(b) $\Omega_{2}=2 \pi \times 1.46 \mathrm{MHz}$

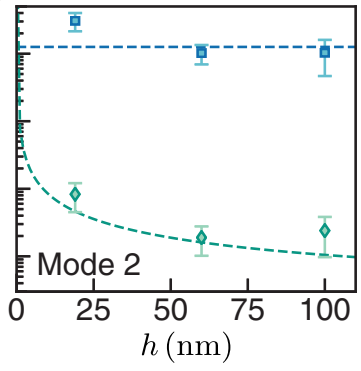

(c) $\Omega_{3}=2 \pi \times 1.525 \mathrm{MHz}$

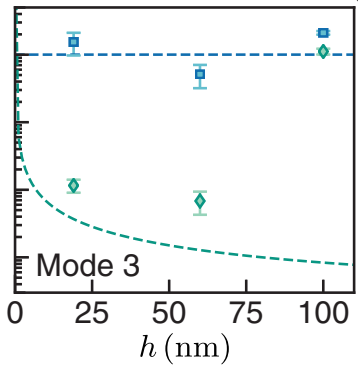

(d) $\Omega_{4}=2 \pi \times 1.527 \mathrm{MHz}$

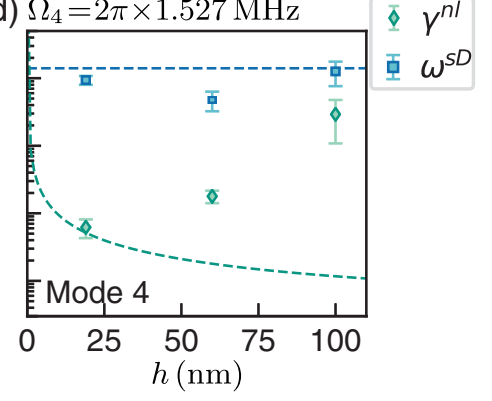

FIG. 2. Nonlinear parameters. (a)-(d) Measured nonlinear parameters as a function of membrane thickness $h$. Blue (green) points are the Duffing (nonlinear damping) parameters $\omega_{i}^{\mathrm{sD}}\left(\gamma_{i}^{\mathrm{nl}}\right)$, estimated from the median of each statistical ensemble. Dashed lines represent simulated values for the Duffing (blue) and nonlinear damping (green) parameters.

[34]. The measurement protocol stated above is repeated for membranes of different thickness, with all nonlinear parameters reported in Fig. 2. (The values obtained for mode 3 of two membranes were discarded since a negative Duffing shift was observed).

We simulate the parameters according to Eqs. (5b) and (5d), where the transverse profile of each mode is obtained by FEM simulations. For modes 1 and 2 we find very good agreement for all investigated thicknesses in the range 19-100 nm. For thicker membranes, we observe excess nonlinear damping in modes 3 and 4, though the Duffing nonlinearity still matches predictions. Possible origins of this deviation are discussed below.

By comparing nonlinear and linear losses experimentally, we now examine the hypothesis of Eq. (9). In Fig. 3, the measured linear quality factors $Q_{\text {meas }}$ are plotted against extracted $\theta_{\mathrm{nl}}^{-1}$. Per our hypothesis, the measured quality factor cannot exceed the function $Q_{\text {meas }} \stackrel{!}{=} D_{Q} \theta_{\mathrm{nl}}^{-1}$, which follows from Eq. (7) if Eq. (9) is correct. The dilution factor for each mode is obtained from FEM simulations, as described earlier [16]. This relation should hold independent of material quality, which may vary between fabrication runs.

Linear loss angles of silicon nitride thin films have been extracted from a large number of resonator data reported in the literature [35]. The expected range is represented by the gray area, where the gray line is the average value. If the hypothesis (9) holds, $\theta_{\mathrm{nl}}^{-1}$ (abscissa in Fig. 3) should also fall in this range.

Although each point should ideally fall between the two lines, additional losses can be introduced during fabrication and handling. The data points would then have larger loss angle but still lie on the oblique line. On the other hand, imperfect dissipation dilution (e.g., damaged structures, residual gas damping, or radiation loss) would lead to data points lying below the oblique line. The regions not explained by these mechanisms are shown as hatched. We observe that the vast majority of the measurements are within the expected region. For the first two modes, most points lie close to the intersection, corroborating our hypothesis. The points' location with respect to the intersection then gives an indication of possible imperfections in the sample.

Nonlinear loss angle measurements for different thicknesses are shown in Figs. 3(e)-3(h). We compare this to the phenomenological model of the linear loss angle as a function of membrane thickness

$$
\theta_{\text {lin }}(h)=Q_{\text {intr }}^{-1}(h)=Q_{\mathrm{vol}}^{-1}+\left(\beta_{s} h\right)^{-1},
$$

where $\quad Q_{\mathrm{vol}}=(2.8 \pm 0.2) \times 10^{4} \quad$ and $\quad \beta_{s}=(60 \pm$ 40) $\mathrm{nm}^{-1}$ [35]. Measured $\theta_{\mathrm{nl}}$ for modes 1 and 2 agree with the model for the $\theta_{\text {lin }}$, within error, supporting our hypothesis. However, the data for modes 3 and 4 show an evident deviation for larger thickness. This is consistent with the excess nonlinear damping observed in Figs. 2(c) and 2(d). The source of this excess nonlinear damping is unclear. Since the isolation provided by the phononic shield for these two modes is, in general, worse [16], we speculate that this can lead to nonlinear energy exchange mediated by vibrational modes of the supporting silicon frame [36].

Lastly, we characterize the intrinsic losses as a function of temperature (cf. Fig. 4). Ringdown measurements were performed on mode 1 of a 19 -nm-thick membrane inside a dilution refrigerator, at temperatures ranging from $20 \mathrm{mK}$ to $1 \mathrm{~K}$. We use $100 \mathrm{nW}$ of optical power at $830 \mathrm{~nm}$ impinging on the membrane, to minimize heating from absorption of optical radiation [37]. An additional reference measurement is taken at room temperature. As reported previously [37-40], we observe an increase of linear quality factor with decreasing temperature. We find $\theta_{\mathrm{nl}}$ decreases in unison with the linear loss over nearly four orders of magnitude span in temperature, in line with our hypothesis. Our new analysis also gives two insights of potential use for further experimental optimization: (a) saturation of the decrease in $\theta_{\mathrm{nl}}$ at around $100 \mathrm{mK}$ suggests that the sample might not thermalize properly to lower temperatures, and (b) near $10^{9}$ the measured linear $Q$ factors stop following $\theta_{\mathrm{nl}}$, suggesting the presence of additional linear, undiluted dissipation. 

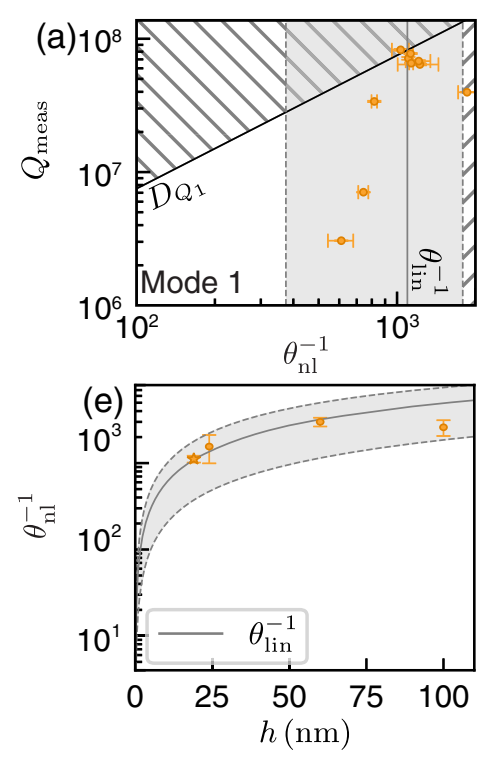
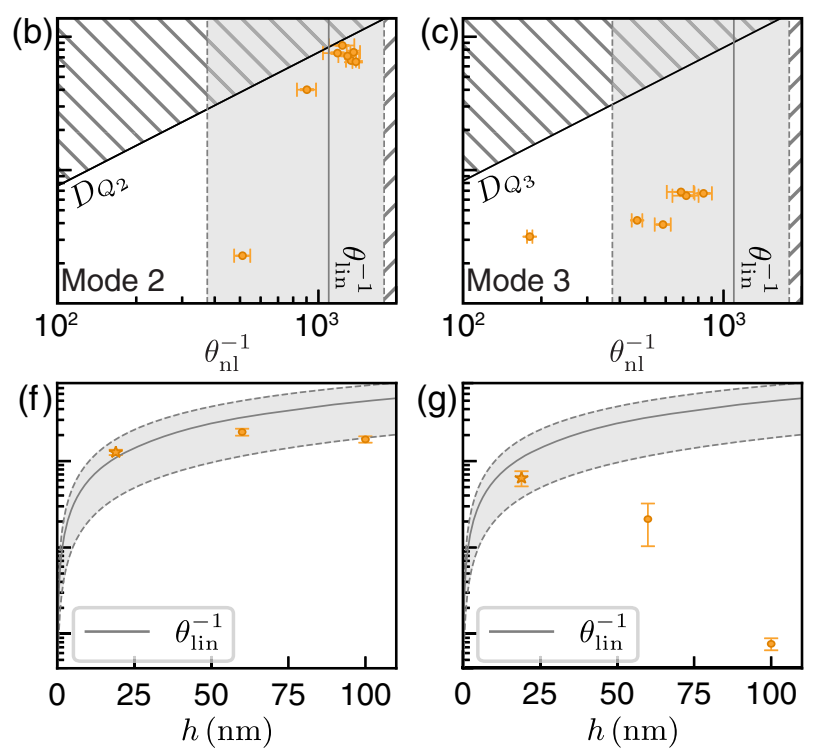
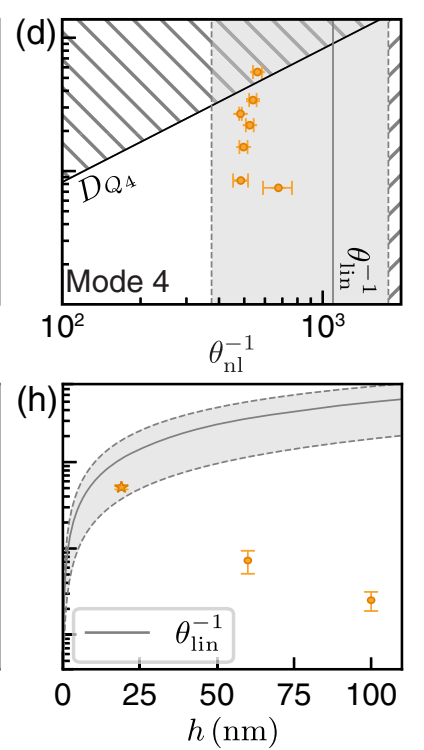

FIG. 3. Nonlinear loss angles. (a)-(d) Measured quality factors against $\theta_{\mathrm{nl}}^{-1}$. The gray line is the expected $\theta_{\operatorname{lin}}^{-1}[35]$ and gray area is the uncertainty in that value. The black line is the limit $Q_{\text {meas }} \leq D_{Q} \theta_{\operatorname{lin}}^{-1}$ with a dissipation dilution factor $D_{Q}$ obtained from simulations. The hatched area is inaccessible under the most obvious sources of excess dissipation. (e)-(h) Measured $\theta_{\mathrm{nl}}^{-1}$ as a function of the membrane thickness. The stars represent the median of the points shown in (a)-(d). The gray line is the $\theta_{\operatorname{lin}}^{-1}$ as a function of thickness, expressed in Eq. (12), whereas the gray area reflects the uncertainty in the parameters.

Conclusion.-Our Letter sheds light on the origin of nonlinear damping in dissipation-diluted nanomechanical resonators. We have developed an analytic theory based on a continuum elastic model for large deflections of a thin membrane. The geometric nonlinearity arising from the material elongation modifies both the conservative and dissipative dynamics, in the form of Duffing frequency shifts and a nonlinear damping. We observe these nonlinear effects in soft-clamped, ultracoherent membrane resonators and find good agreement with our model.
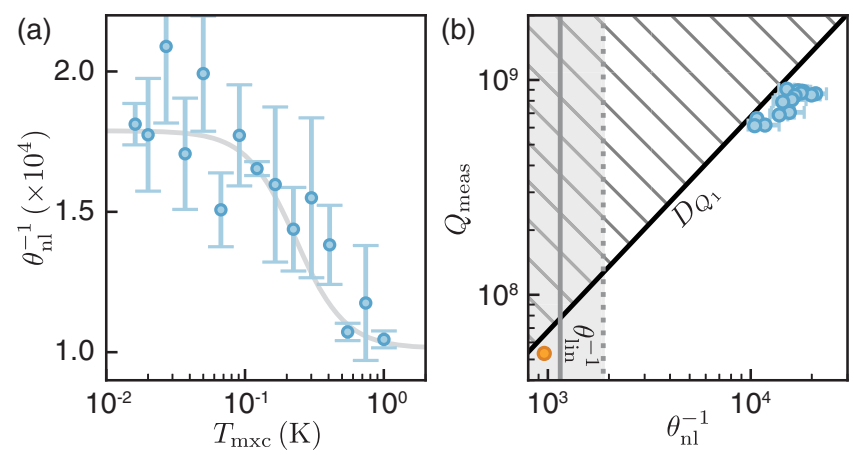

FIG. 4. Nonlinear loss angles at different temperatures. (a) Measured $\theta_{\mathrm{nl}}^{-1}$ as a function of the cryostat temperature $T_{\mathrm{mxc}}$. The gray line is a polynomial fit, roughly showing the behavior. (b) Measured quality factors versus $\theta_{\mathrm{nl}}^{-1}$, taken at room temperature (orange) and cryogenic temperatures (blue). The gray line is the expectation value of $\theta_{\text {lin }}^{-1}$ at room temperature, and the gray area reflects uncertainty [35]. The black line is the simulated quality factor, from the dissipation dilution factor $D_{Q_{1}}$. Error bars are the mean absolute deviation among three repetitions.
We introduce the nonlinear loss angle $\theta_{\mathrm{nl}}$ and show that it can be extracted from ringdown measurements without displacement calibration. Our model hypothesizes $\theta_{\mathrm{nl}}$ is equal to the linear loss angle, which is otherwise not separately accessible by measurement. We find substantial evidence supporting this hypothesis across a wide array of mode shapes, geometric parameters, and temperatures. These insights deepen our understanding of nonlinear behavior in this important class of nanomechanical resonators and can guide design of future generations of ultracoherent mechanical sensors [16-18], especially with regard to sensing protocols [26]. Finally, the tools developed here yield additional insight in the performance and loss contributions of dissipation-diluted resonators.

The authors acknowledge Y. Tsaturyan for sample fabrication and $\mathrm{D}$. Mason for assistance with the interferometric nonlinear transduction. This work was supported by the Swiss National Science Foundation (Grant No. 177198), the European Research Council project Q-CEOM (Grant No. 638765), the Danish National Research Foundation (Center of Excellence "Hy-Q"), the EU H2020 FET proactive project HOT (Grant No. 732894), and the Novo Nordisk Foundation (Grant No. NNF20OC0061866).

*Present address: Photonics Laboratory, ETH Zürich, 8093 Zürich, Switzerland. †albert.schliesser@nbi.ku.dk 
[1] A. N. Cleland, Foundations of Nanomechanics (Springer, Berlin, Heidelberg, 2003).

[2] S. Schmid, L. G. Villanueva, and M. L. Roukes, Fundamentals of Nanomechanical Resonators (Springer International Publishing, New York, 2016).

[3] C. Reinhardt, T. Müller, A. Bourassa, and J. C. Sankey, Ultralow-Noise SiN Trampoline Resonators for Sensing and Optomechanics, Phys. Rev. X 6, 021001 (2016).

[4] J. Chaste, A. Eichler, J. Moser, G. Ceballos, R. Rurali, and A. Bachtold, A nanomechanical mass sensor with yoctogram resolution, Nat. Nanotechnol. 7, 301 (2012).

[5] F. Yi, H. Zhu, J. C. Reed, and E. Cubukcu, Plasmonically enhanced thermomechanical detection of infrared radiation, Nano Lett. 13, 1638 (2013).

[6] M.-H. Chien, M. Brameshuber, B. K. Rossboth, G. J. Schütz, and S. Schmid, Single-molecule optical absorption imaging by nanomechanical photothermal sensing, Proc. Natl. Acad. Sci. U.S.A. 115, 11150 (2018).

[7] L. Midolo, A. Schliesser, and A. Fiore, Nano-opto-electromechanical systems, Nat. Nanotechnol. 13, 11 (2018).

[8] G. Kurizki, P. Bertet, Y. Kubo, K. Mølmer, D. Petrosyan, P. Rabl, and J. Schmiedmayer, Quantum technologies with hybrid systems, Proc. Natl. Acad. Sci. U.S.A. 112, 3866 (2015).

[9] M. Pechal, P. Arrangoiz-Arriola, and A. H. Safavi-Naeini, Superconducting circuit quantum computing with nanomechanical resonators as storage, Quantum Sci. Technol. 4, 015006 (2018).

[10] G. I. González and P. R. Saulson, Brownian motion of a mass suspended by an anelastic wire, J. Acoust. Soc. Am. 96, 207 (1994).

[11] Y. L. Huang and P. R. Saulson, Dissipation mechanisms in pendulums and their implications for gravitational wave interferometers, Rev. Sci. Instrum. 69, 544 (1998).

[12] S. S. Verbridge, D. F. Shapiro, H. G. Craighead, and J. M. Parpia, Macroscopic tuning of nanomechanics: Substrate bending for reversible control of frequency and quality factor of nanostring resonators, Nano Lett. 7, 1728 (2007).

[13] Q. P. Unterreithmeier, T. Faust, and J. P. Kotthaus, Damping of Nanomechanical Resonators, Phys. Rev. Lett. 105, 027205 (2010).

[14] S. Schmid, K. D. Jensen, K. H. Nielsen, and A. Boisen, Damping mechanisms in high-qmicro and nanomechanical string resonators, Phys. Rev. B 84, 165307 (2011).

[15] S. A. Fedorov, N. J. Engelsen, A. H. Ghadimi, M. J. Bereyhi, R. Schilling, D. J. Wilson, and T. J. Kippenberg, Generalized dissipation dilution in strained mechanical resonators, Phys. Rev. B 99, 054107 (2019).

[16] Y. Tsaturyan, A. Barg, E. S. Polzik, and A. Schliesser, Ultracoherent nanomechanical resonators via soft clamping and dissipation dilution, Nat. Nanotechnol. 12, 776 (2017).

[17] A. H. Ghadimi, S. A. Fedorov, N. J. Engelsen, M. J. Bereyhi, R. Schilling, D. J. Wilson, and T. J. Kippenberg, Elastic strain engineering for ultralow mechanical dissipation, Science 360, 764 (2018).

[18] C. Reetz, R. Fischer, G. G. P. Assumpção, D. P. McNally, P. S. Burns, J. C. Sankey, and C. A. Regal, Analysis of Membrane Phononic Crystals with Wide Band Gaps and Low-Mass Defects, Phys. Rev. Applied 12, 044027 (2019).
[19] L. Catalini, Y. Tsaturyan, and A. Schliesser, Soft-Clamped Phononic Dimers for Mechanical Sensing and Transduction, Phys. Rev. Applied 14, 014041 (2020).

[20] M. Imboden, O. A. Williams, and P. Mohanty, Observation of nonlinear dissipation in piezoresistive diamond nanomechanical resonators by heterodyne down-mixing, Nano Lett. 13, 4014 (2013).

[21] A. Eichler, J. Moser, J. Chaste, M. Zdrojek, I. Wilson-Rae, and A. Bachtold, Nonlinear damping in mechanical resonators made from carbon nanotubes and graphene, Nat. Nanotechnol. 6, 339 (2011).

[22] K. Y. Fong, W. H. P. Pernice, and H. X. Tang, Frequency and phase noise of ultra-high $\mathrm{Q}$ silicon nitride nanomechanical resonators, Phys. Rev. B 85, 161410(R) (2012).

[23] F. Hocke, M. Pernpeintner, X. Zhou, A. Schliesser, T. J. Kippenberg, H. Huebl, and R. Gross, Determination of effective mechanical properties of a double-layer beam by means of a nano-electromechanical transducer, Appl. Phys. Lett. 105, 133102 (2014).

[24] M. Defoort, K. J. Lulla, C. Blanc, H. Ftouni, O. Bourgeois, and E. Collin, Stressed silicon nitride nanomechanical resonators at helium temperatures, J. Low Temp. Phys. 171, 731 (2013).

[25] S. Zaitsev, O. Shtempluck, E. Buks, and O. Gottlieb, Nonlinear damping in a micromechanical oscillator, Nonlinear Dyn. 67, 859 (2012).

[26] J. Košata, O. Zilberberg, C. L. Degen, R. Chitra, and A. Eichler, Spin Detection via Parametric Frequency Conversion in a Membrane Resonator, Phys. Rev. Applied 14, 014042 (2020).

[27] L. D. Landau and E. M. Lifshitz, Theory of Elasticity (Pergamon Press, New York, 1970).

[28] J. Atalaya, A. Isacsson, and J. M. Kinaret, Continuum elastic modeling of graphene resonators, Nano Lett. 8, 4196 (2008).

[29] M. I. Younis, MEMS Linear and Nonlinear Statics and Dynamics (Springer, New York, 2011).

[30] P. M. Polunin, Y. Yang, M. I. Dykman, T. W. Kenny, and S. W. Shaw, Characterization of MEMS resonator nonlinearities using the ringdown response, J. Microelectromech. Syst. 25, 297 (2016).

[31] T. Antoni, K. Makles, R. Braive, T. Briant, P.-F. Cohadon, I. Sagnes, I. Robert-Philip, and A. Heidmann, Nonlinear mechanics with suspended nanomembranes, Europhys. Lett. 100, 68005 (2012).

[32] A. Gusso, Nonlinear damping in suspended beam microand nanoresonators due to surface loss, J. Sound Vib. 467, 115067 (2020).

[33] See Supplemental Material at http://link.aps.org/ supplemental/10.1103/PhysRevLett.126.174101 for detailed mathematical derivations, calibration and experimental methods, modeshape of the modes of interest, and complete set of measured nonlinear loss angles.

[34] T. Pham-Gia and T. Hung, The mean and median absolute deviations, Math. Comput. Modell. 34, 921 (2001).

[35] L. G. Villanueva and S. Schmid, Evidence of Surface Loss as Ubiquitous Limiting Damping Mechanism in SiN Microand Nanomechanical Resonators, Phys. Rev. Lett. 113, 227201 (2014). 
[36] Y. S. Patil, S. Chakram, L. Chang, and M. Vengalattore, Thermomechanical Two-Mode Squeezing in an UltrahighQ Membrane Resonator, Phys. Rev. Lett. 115, 017202 (2015).

[37] M. A. Page, M. Goryachev, H. Miao, Y. Chen, Y. Ma, D. Mason, M. Rossi, C. D. Blair, L. Ju, D. G. Blair, A. Schliesser, M. E. Tobar, and C. Zhao, Gravitational wave detectors with broadband high frequency sensitivity, Commun. Phys. 4, 27 (2021).

[38] M. Yuan, M. A. Cohen, and G. A. Steele, Silicon nitride membrane resonators at millikelvin temperatures with quality factors exceeding $10^{8}$, Appl. Phys. Lett. 107, 263501 (2015).

[39] T. Faust, J. Rieger, M. J. Seitner, J. P. Kotthaus, and E. M. Weig, Signatures of two-level defects in the temperature-dependent damping of nanomechanical silicon nitride resonators, Phys. Rev. B 89, 100102(R) (2014).

[40] R. Fischer, N. S. Kampel, G. G. T. Assumpção, P.-L. Yu, K. Cicak, R. W. Peterson, R. W. Simmonds, and C. A. Regal, Optical probing of mechanical loss of a $\mathrm{Si}_{3} \mathrm{~N}_{4}$ membrane below $100 \mathrm{mK}$, arXiv:1611.00878. 